\title{
In vitro Fertilization with Mouse Sperm Activated by Components of Licorice Root Extract
}

\author{
Nguyen Huu Tung ${ }^{1,4}$, Hiromitsu Tanaka ${ }^{1}$, Akira Tsujimura², Yasushi Miyagawa ${ }^{3}$, Morimasa Wada1, Shunsuke Fujii', Takuhiro Uto and Yukihiro \\ Shoyama ${ }^{1 *}$ \\ ${ }^{1}$ Faculty of Pharmaceutical Sciences, Nagasaki International University, 2825-7 Huis Ten Bosch, Sasebo, Nagasaki 859-3298, Japan \\ ${ }^{2}$ Department of Urology, Juntendo University Hospital, Japan \\ ${ }^{3}$ Department of Urology, Graduate School of Medicine, Osaka University, Japan
}

${ }^{4}$ School of Medicine and Pharmacy, Vietnam National University, Hanoi, Vietnam

\begin{abstract}
Licorice (Glycyrrhiza species) is an important natural product that is frequently used in traditional Chinese medicine. In this review article, we discuss the quality control of licorice using monoclonal antibodies against the licorice components glyzyrrhizin (GC) and liquiritin (Liq); the monitoring of licorice extract activity using an assay system in mice; and the isolation of two active phenolic components, isolitiritigenin and formononetin, and their effectiveness for improving in vitro fertilization in mice. Finally, we present the outstanding questions and potential applications of licorice root extract.
\end{abstract}

Keywords: Licorice; Glycyrrhiza species; Isoliquiritigenin; Hormononetin; In vitro fertilization; Mice

\section{Introduction}

Glycyrrhiza species (Leguminosae), commonly known as licorice root are perennial plants that grow up to $1.5 \mathrm{~m}$ high and are distributed in drylands from Western Europe to Russia, and are particularly abundant in China and Mongolia. G. uralensis Fisch (Figure 1) and G. glabra are listed in the Japanese pharmacopoeia.

Nearly 500 compounds have been identified in licorice root; including glycyrrhizin (GC)-which is controlled at a concentration of more than $2.5 \%$ and the flavonoid liquiritin (Liq) has a quantitative limitation in Japanese Pharmacopoeia [1]. Flavonoids have antihepatotoxic, anti-inflammatory, anti-ulcer, anti-allergenic, and antiviral as well as cardioprotective activities [2]; owing to these medicinal properties, licorice root is used in at least $70 \%$ of traditional Chinese medicines for treatment of nervous, alimentary, respiratory, endocrine, and cardiovascular system diseases [3]. Licorice is also widely used as a sweetener in the production of confectioneries and soy sauce and is therefore in high demand worldwide. Using a Monoclonal Antibody (MAb) against GC prepared in our laboratory [4], we previously reported on the synergistic effects of the major constituent of licorice including GC and other constituents [5], the interfacial behavior of GC [6], and the identification of Glycyrrhiza species with higher GC content [7].

Artificial insemination is an assisted reproductive technology in which pregnancy is achieved by artificially introducing semen into the female genital tract; it is an indispensable technology for breeding cattle and other livestock and is used for infertility treatment in humans [8]. An in vitro Fertilization (IVF) system has been developed in mice, and many mouse lines have been produced by this method by freezing embryos and/or fertilized eggs [9]. However, IVF using sperm that is frozen or refrigerated over a long term would allow mouse lines to be preserved more easily. Sperm cells start moving immediately after ejaculation and undergo maturation after a defined period of time (capacitation), causing an acrosome reaction that leads to fertilization of an egg. However, the efficiency of IVF is relatively low. It is known that several traditional Japanese medicines (TJM) like Tokishakuyakusan for female infertility [10] and Hochuekkito for male infertility [11]. Furthermore, Traditional Chinese herbal medicine have been searched widely for female infertility $[12,13]$. We previously investigated the effects of crude licorice extract on the IVF rate [14] and isolated two active components of licorice that may be responsible for these effects [15]. In this review, we describe the active components of licorice extract, their isolation, and potential applications for improving IVF rates.

\section{Components of Licorice and Quantitative and Qualitative Control}

Licorice is listed in Material Medica and Shennong Ben Cao Jing as an important and safe medicine. Over 500 compounds have been identified in licorice root including those of the triterpene saponin group like GC and two types of flavonoid including isoflabonoid (Figure 2).

GC and Liq are two major components in licorice that have wellknown pharmacological activities [2]. Quality control of licorice root is needed to assure uniformity in the concentrations of these compounds, which can differ according to licorice species, growth place, and harvest season, and so on. To this end, we previously prepared MAbs against GC [4] and Liq [16] and developed an eastern blotting system for detection of GC and Liq and their aglycones (glycyrrhetinic acid and liquiritigenin, respectively) [17] in order to make evident the resource of licorice for further researches. In this method, crude licorice extract and/or traditional Chinese medicine extract are developed on a Thin

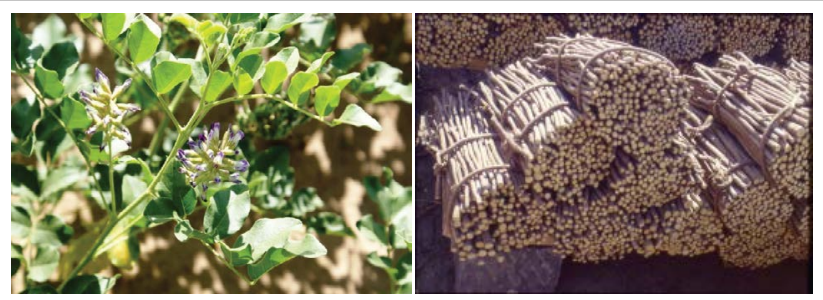

Figure 1: Flowering of Glycyrrhiza uralensis Fisch and its crude drug

*Corresponding author: Yukihiro Shoyama, Faculty of Pharmaceutical Sciences, Nagasaki International University, 2825-7 Huis Ten Bosch, Sasebo, Nagasaki 8593298, Japan, Tel: +81956205622; E-mail: shoyama@niu.ac.jp

Received March 08, 2016; Accepted April 18, 2016; Published April 24, 2016

Citation: Tung NH, Tanaka H, Tsujimura A, Miyagawa Y, Wada M, et al. (2016) In vitro Fertilization with Mouse Sperm Activated by Components of Licorice Root Extract. Nat Prod Chem Res 4: 217. doi:10.4172/2329-6836.1000217

Copyright: ( 2016 Tung NH, et al. This is an open-access article distributed under the terms of the Creative Commons Attribution License, which permits unrestricted use, distribution, and reproduction in any medium, provided the original author and source are credited. 


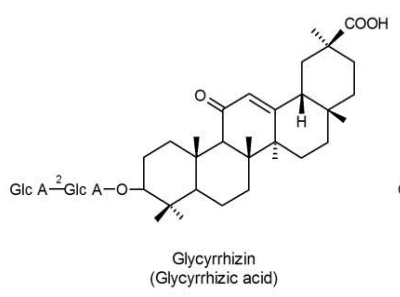<smiles>CC(C)=CCc1cc2c(=O)cc(-c3ccc(O)cc3)oc2cc1O</smiles><smiles>COc1cc2c(c(O)c1CC=C(C)C)CC(c1ccc(O)c(CC=C(C)C)c1O)CO2</smiles>

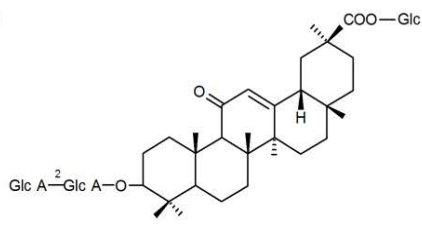

Licorice-saponin A3<smiles>CC(C)=CCc1c(O)cc(O)c(-c2coc3cc(O)ccc3c2=O)c1O</smiles>

Licoricone

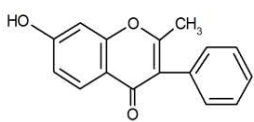

2-Methyl-7-hydroxyisoflavone<smiles>COc1ccc(C2CC(=O)c3ccc(O)cc3O2)cc1</smiles>

Liquiritin<smiles></smiles>

Glycyrol<smiles>CC1(C)C=Cc2c(ccc(C3=Cc4ccc(O)cc4OC3)c2O)O1</smiles><smiles>Cc1cc(=O)oc2ccc(O)c(O)c12</smiles><smiles>COc1ccc(/C=C/C(=O)c2ccc(O)cc2O)cc1</smiles>

Isoliquiritin<smiles>COc1c2c(cc3oc(=O)c4c5ccc(O)cc5oc4c13)OC(C)(C)CC2</smiles>

Isoglycyrol

Liqcoumarin

Figure 2: Major components of licorice root.

Layer Chromatography (TLC) plate and transferred to a membrane by heating (Figure 3). The membrane is treated with sodium periodide, yielding an aldehyde group that can be conjugated with a carrier protein that is then bound to the membrane. GC and Liq on the membrane can be detected by labeling with anti-GC and anti-Liq MAbs (Figure 3B). However, two aglycones cannot be labeled simultaneously by eastern blotting (Figure 3B) because unlike TLC (Figure 3A), there are no sugar moieties that can be conjugated to the membrane. This method ensures quality control of licorice root sources by analysis of the two major components.

\section{Activity of Crude Licorice Extract}

The capacity for fertilization of sperm from wild-type C57BL/6 mice was high using standard (e.g., HTF) medium supplemented with bovine serum albumin for IVF or with polyvinyl alcohol (PVA) plus methyl $\beta$-cyclodextrin (MBCD). When the sperm of aged BALB/cA mice ( $>48$ weeks of age) was used [18], IVF efficiency was low, with fertilization capacity varying between mice. However, the fertilization rate for HTF medium containing PVA plus MBCD and licorice extract $(0.12 \mathrm{mg} / \mathrm{ml})$ was between $15.6 \%$ and $84.0 \%$ as compared to $0.0 \%$ using medium containing $0.25 \%$ DMSO. The optimal concentration of licorice extract in HTF medium was $0.3 \mathrm{mg} / \mathrm{ml}$.

Although ingesting an excess of GC can cause steroid hormone-like activity and an allergic reaction to licorice, it is generally safe and widely used as a sweetener. There are no reports of licorice increasing pregnancy rates [19]; however, licorice extract improved the fertilization capacity of $\mathrm{BALB} / \mathrm{cA}$ mouse sperm in vitro and fertilized eggs developed normally. In rats, licorice extract increased cyclophosphamide teratogenicity and upregulated the mRNA expression of cytochrome P-4502B [20], suggesting that licorice root contains multiple components with biological activity that may not necessarily affect gene expression, given that transcription rates are low in spermatozoa [21].

The presence of GC alone in the medium did not improve fertilization rates, implying that other substances are involved. Licorice extracts preserved sperm mobility without inducing the acrosome reaction in sperm. Hence, it is expected that distinct signals are responsible for the acrosome reaction and sperm motility. Some transgenic mouse lines show very poor IVF efficiency [22]; as such, it would be useful to improve IVF culture medium by including factors that can enhance fertilization rates. While the results described above in mice are promising, it remains to be determined whether licorice root extract can improve IVF rates in other animals, including humans.

\section{Identification of Active Compounds for IVF}

An air-dried sample of $G$. uralensis root was sliced and then extracted with hot distilled water under sonication. The combined extracts were filtered and concentrated under reduced pressure, and the obtained residue was lyophilized to produce a crude aqueous licorice extract that was resuspended in water and then successively partitioned with $\mathrm{EtOAc}$ and $\mathrm{n}-\mathrm{BuOH}$. Each fraction was evaporated under reduced pressure, leaving only residues. The EtOAc fraction showed the strongest activity, as evidenced by fertilization rates (Figure 4).

The EtOAc fraction was separated by silica gel column chromatography with a $\mathrm{CHCl}_{3}-\mathrm{MeOH}$ solvent system as the eluent, yielding five sub-fractions (1-5). Fraction 2 was further separated using a reversed-phase $\mathrm{C} 18$ column with $\mathrm{MeOH}-\mathrm{H}_{2} \mathrm{O}$, yielding active compound 1. Similarly, active compound 2 was purified from fraction 4 using a reversed-phase $\mathrm{C} 18$ column with $\mathrm{MeOH}-\mathrm{H}_{2} \mathrm{O}(1: 1, \mathrm{v} / \mathrm{v})$.

Compound 1 was a yellow powder that showed an ion peak at $\mathrm{m} / \mathrm{z}$ $257[\mathrm{M}+\mathrm{H}]^{+}$and strong yellow fluorescence, suggesting a chalcone derivative; this was confirmed by ${ }^{13} \mathrm{C}$ Nuclear Magnetic Resonance (NMR). By 1-dimensional (1D; ${ }^{1} \mathrm{H}$ and ${ }^{13} \mathrm{C}$ ) and 2D correlation spectroscopy (COSY) and heteronuclear multiple quantum coherence (HMQC) and Heteronuclear Multiple-Bond Coherence (HMBC) spectroscopy, it was determined that compound $\mathbf{1}$ was isoliquiritigenin [23].

Compound 2 was a pale yellow powder that showed an ion peak at $\mathrm{m} / \mathrm{z} 268[\mathrm{M}+\mathrm{H}]^{+}$; its mass fragment pattern suggested a flavonoid compound with a methoxyl group. ${ }^{13} \mathrm{C}$ NMR revealed a typical lowershifted C-3 carbon, suggesting that compound 2 was an isoflavone; $1 \mathrm{D}$ 


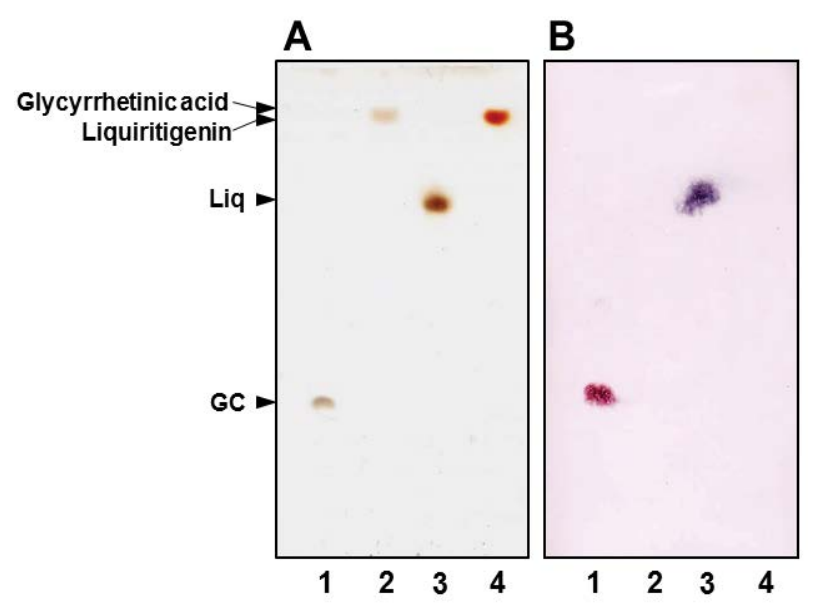

Figure 3: Detection of GC, glycyrrhetinic acid, Liq, and liquiritigenin by sulfuric acid staining $(A)$ and double eastern blotting using anti-GC and anti-Liq MAbs (B). Lanes 1-4 represent GC, glycyrrhetinic acid, Liq, and liquiritigenin. The TLC plate was developed with $\mathrm{n}-\mathrm{BuOH} /$ water/acetic acid (7/2/1, v/v/v).

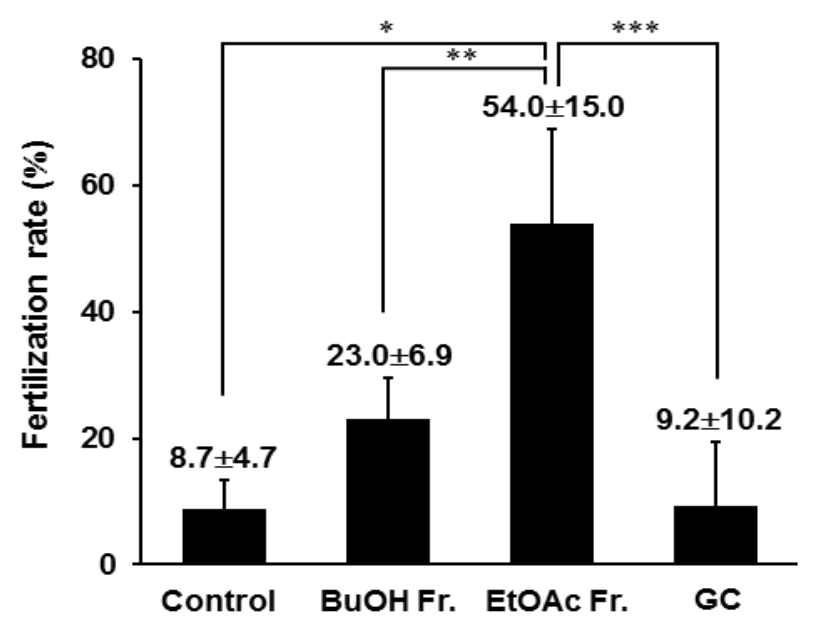

Figure 4: IVF rates using active fractions of licorice extract and GC. n-BuOH, EtOAc fractions and GC were added to the conditioned medium [14]. Numbers indicate mean $\pm \mathrm{SE}$. ${ }^{\mathrm{P}} \mathrm{P}<0.05,{ }^{\mathrm{*}} \mathrm{P}<0.01,{ }^{* * *} \mathrm{P}<0.001$.

$\left({ }^{1} \mathrm{H}\right.$ and $\left.{ }^{13} \mathrm{C}\right)$ and 2D (COSY, HMQC, and HMBC) spectra confirmed that compound 2 was formononetin [24] (Figure 5).

\section{Activity of Isoliquiritingenin and Formononetin}

We investigated the effects of isoliquiritingenin and formononetin on sperm and found that the fertilization rates of HTF medium containing PVA plus $\mathrm{MBCD}$ and either isoliquiritigenin or formononetin at a concentrations ranging from $0-0.04 \mathrm{mg} / \mathrm{ml}$ were as high as $47.2 \% \pm 16.8 \%$ for isoliquiritingenin and $50.2 \% \pm 9.8 \%$ for formononetin (Figure 6).

We examined the viability of embryos treated with isoliquiritigenin or formononetin and found that the embryos were morphologically normal (Figure 7), consistent with previous observations using licorice crude extract [14].

\section{Outstanding Questions and Future Directions}

Artificial insemination is widely used in livestock breading and for fertility treatment in humans, while genetically modified mice have been generated to evaluate gene function in vivo. Given their importance as research materials, an effective method for preserving various transgenic mice is needed, especially given the low efficiency of IVF in some lines. We previously showed that fertilization rate was improved using aqueous licorice extract in $\mathrm{BALB} / \mathrm{c}$ mice, which have a low fertilization rate. The licorice extracts stimulated fertilization without inducing a spontaneous acrosome reaction in the sperm or causing developmental defects.

The signals that contribute to the maturation of ejaculated sperm remain largely unknown. Estrogen was reported to modulate sperm activation and the acrosome reaction [25]. We identified two active components, formononetin and isoliquiritigenin, in licorice root that promote IVF [15]. A study of three licorice species (G. uralensis, G. glabra, and G. inflata) found that isoliquiritigenin had strong estrogenlike activity, suggesting that it is cyclized to liquiritigenin, an active flavonoid, under physiological conditions [26]. We also found that the hydrolysis of diarylheptanoid glycoside to free diarylheptanoid in culture medium has anti-trypanosomal and apoptotic activities [27], suggesting that isoliquiritigenin is a liquiritigenin precursor. In fact the biosynthetic pathway between isoliquiritigenin to formononetin via liquiritigenin starting from cinnamic acid was confirmed using microorgainisms and G. echinata cell-free system, respectively as shown in Figure $8[28,29]$.

Hormononetin, an isoflavone are implicated in various aspects of sexual development, including pubertal timing, estrous cycling, and ovarian, hypothalamus, and pituitary functions [30]. Since many flavonoids including isoflavone are biosynthesized on the above pathway, it is supposed that other active flavonoid may be able to be found out. Although the relationship between estrogen and fertilization is not entirely clear, the two phytoestrogens described here may promote fertilization. It is also possible that some of the 500 components of licorice act synergistically to stimulate fertilization.

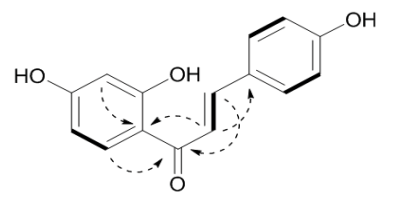

Isoliquiritingenin

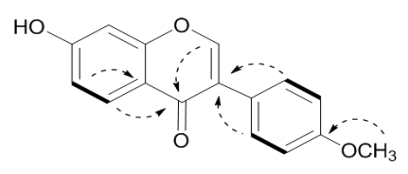

Formononetin
Figure 5: Chemical structures of isoliquiritingenin and formononetin, as determined by COSY and HMBC. Arrows with broken lines represent the movement of electrons.

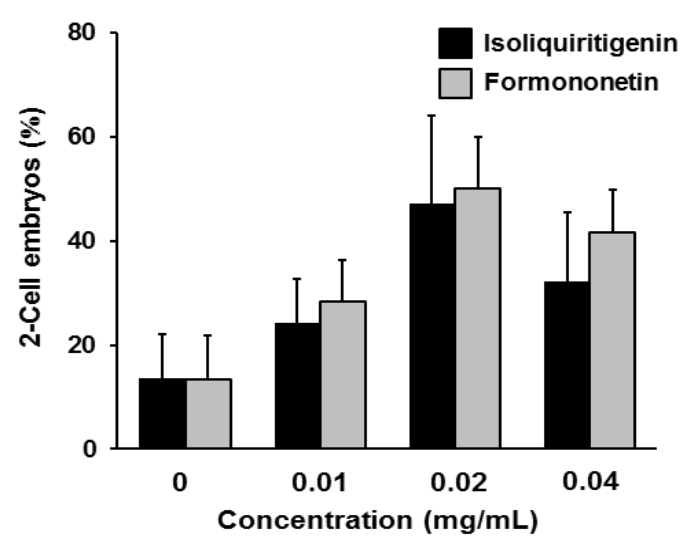

Figure 6: Optimal concentration of licorice extract for IVF. Data are presented as mean $\pm 95 \%$ confidence intervals of five experiments. 
Citation: Tung NH, Tanaka H, Tsujimura A, Miyagawa Y, Wada M, et al. (2016) In vitro Fertilization with Mouse Sperm Activated by Components of Licorice Root Extract. Nat Prod Chem Res 4: 217. doi:10.4172/2329-6836.1000217

Page 4 of 4

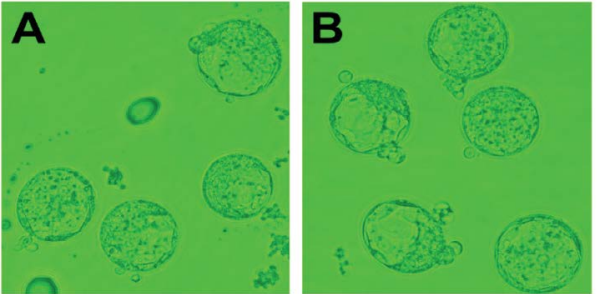

Figure 7: Blastocysts incubated with licorice extract. Embryos treated with isoliquiritigenin (A) or formononetin (B) did not show morphological abnormalities [15], consistent with observations using licorice crude extract [14].

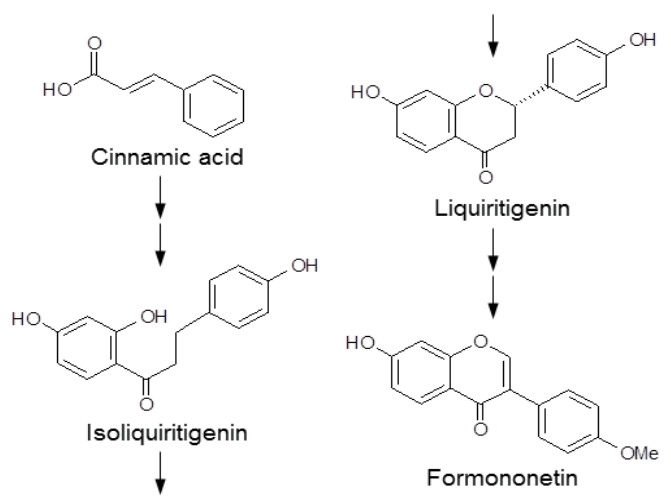

Figure 8: Biosynthetic pathway between isoliquiritigenin to formononetin.

We recently developed a MAb that can be used to purify additional active components from isoliquiritigenin and formononetin knockout extracts [31]. In addition to the various reported functions of isoliquiritigenin-including anti-inflammatory, immunoregulatory, antimicrobial, antioxidant, anticancer, hepatoprotective, and cardioprotective activities [32] both isoliquiritigenin and formononetin have been shown to modulate sperm during fertilization. Clarifying the molecular mechanisms underlying the effects of these compounds on sperm can provide a basis for improving current IVF practices.

\section{References}

1. (2015) Japanese Pharmacopoeia. 16th edn.

2. Agrawal AD (2011) Pharmacological activities of flavonoids: A review. Int $J$ Pharma Sci Nanotech 4: 1394-1398.

3. Yang R, Wang LQ, Yuan BC, Liu Y (2015) The Pharmacological Activities of Licorice. Planta Med 81: 1654-1669.

4. Shan S, Tanaka H, Shoyama $Y$ (2001) Enzyme-linked immunosorbent assay for glycyrrhizin using anti-glycyrrhizin monoclonal antibody and an eastern blotting technique for glucuronides of glycyrrhetic acid. Anal Chem 73: 5784-5790.

5. Uto T, Morinaga O, Tanaka H, Shoyama Y (2012) Analysis of the synergistic effect of glycyrrhizin and other constituents in licorice extract on lipopolysaccharide-induced nitric oxide production using knock-out extract. Biochem Biophys Res Commun 417: 473-478.

6. Sakamoto S, Nakahara H, Uto T, Shoyama Y, Shibata O (2013) Investigation of interfacial behavior of glycyrrhizin with a lipid raft model via a Langmuir monolayer study. Biochim Biophys Acta 1828: 1271-1283.

7. Fujii S, Tuvshintogtokh I, Mandakh B, Munkhjargal B, Uto T, et al. (2014) Screening of Glycyrrhiza uralensis Fisch. ex DC. containing high concentrations of glycyrrhizin by Eastern blotting and enzyme-linked immunosorbent assay using anti-glycyrrhizin monoclonal antibody for selective breeding of licorice. $J$ Nat Med 68: 717-722.

8. Bhattacharya S, Maheshwari A, Mollison J (2013) Factors associated with failed treatment: an analysis of 12,744 women embarking on their first IVF cycles. PLoS One 8: e82249.
9. Toyoda Y, Yokoyama M, Hoshi T (1978) Studies on the fertilization of mouse egg in vitro. Jpn J Anim Reprod 16: 147-151.

10. Kimura T (1984) Clinical fertility example by Tokishakuyakusan. Med J Iwate Pref Hospital 24: 56.

11. Yamamoto M (1993) Modern trends of treatment in male infertility. Nihon Hinyokika Gakkai Zasshi 84: 229-243.

12. Ried K, Stuart K (2011) Efficacy of Traditional Chinese Herbal Medicine in the management of female infertility: a systematic review. Complement Ther Med 19: 319-331.

13. Ried K (2015) Chinese herbal medicine for female infertility: an updated metaanalysis. Complement Ther Med 23: 116-128.

14. Tung NH, Shoyama $\mathrm{Y}$, Wada $\mathrm{M}$, Tanaka $\mathrm{H}$ (2014) Improved in vitro fertilization ability of mouse sperm caused by the addition of licorice extract to the preincubation medium. Open Reprod Sci J 6: 1-7.

15. Tung NH, Shoyama Y, Wada M, Tanaka H (2015) Two activators of in vitro fertilization in mice from licorice. Biochem Biophys Res Commun 467: 447-450.

16. Fujii S, Morinaga O, Uto T, Nomura S, Shoyama Y (2014) Development of a monoclonal antibody-based immunochemical assay for liquiritin and its application to the quality control of licorice products. J Agric Food Chem 62 3377-3383.

17. Fujii S, Morinaga O, Uto T, Nomura S, Shoyama Y (2016) Development of Double Eastern Blotting for Major Licorice Components, Glycyrrhizin and Liquiritin for Chemical Quality Control of Licorice Using anti-Glycyrrhizin and anti-Liquiritin Monoclonal Antibodies. J Agric Food Chem 64: 1087-1093.

18. Vasudevan K, Sztein JM (2011) Treatment of sperm with extracellular adenosine 5 '-triphosphate improves the in vitro fertility rate of inbred and genetically modified mice with low fertility. Theriogenology 76: 729-736.

19. Shin S, Jang JY, Choi BI, Baek IJ, Yon JM, et al. (2008) Licorice extract does not impair the male reproductive function of rats. Exp Anim 57: 11-17.

20. Park D, Yang YH, Choi EK, Yang G, Bae DK, et al. (2011) Licorice extract increases cyclophosphamide teratogenicity by upregulating the expression of cytochrome P-450 2B mRNA. Birth Defects Res B Dev Reprod Toxicol 92 553-559.

21. Tanaka H, Baba T (2005) Gene expression in spermiogenesis. Cell Mol Life Sci 62: 344-354.

22. Yanagimachi $R$ (2009) Germ cell research: a personal perspective. Biol Reprod 80: 204-218.

23. Fu B, Li H, Wang X, Lee FS, Cui S (2005) Isolation and identification of flavonoids in licorice and a study of their inhibitory effects on tyrosinase. J Agric Food Chem 53: 7408-7414.

24. Yankep E, Fomum ZT, Bisra D, Dagne E, Hellwig V, et al. (1998) O-Geranylated isoflavones and a3-phenylcoumarin from Millettia griffoniana. Phytochemistry 49: 2521-2523

25. Bathla H, Guraya SS, Sangha GK (1999) Role of estradiol in the capacitation and acrosome reaction of hamster epididymal spermatozoa in the isolated uterus of mice incubated in vitro. Indian J Physiol Pharmacol 43: 211-217.

26. Hajirahimkhan A, Simmler C, Yuan Y, Anderson JR, Chen SN, et al. (2013) Evaluation of estrogenic activity of licorice species in comparison with hops used in botanicals for menopausal symptoms. PLoS One 8: e67947.

27. Uto T, Tung NH, Appiah-Opong R, Aning A, Morinaga O, et al. (2015) Antiproliferative and Pro-Apoptotic Activity of Diarylheptanoids Isolated from the Bark of Alnus japonica in Human Leukemia Cell Lines. Am J Chin Med 43 757-767.

28. Yan Y, Huang L, Koffas MA (2007) Biosynthesis of 5-deoxyflavanones in microorganisms. Biotechnol J 2: 1250-1262.

29. Akashi T, Sawada Y, Aoki T, Ayabe S (2000) New scheme of the biosynthesis of formononetin involving 2,7,4'-trihydroxyisoflavanone but not daidzein as the methyl acceptor. Biosci Biotechnol Biochem 64: 2276-2279.

30. Kim SH, Park MJ (2012) Effects of phytoestrogen on sexual development Korean J Pediatr 55: 265-271.

31. Uto T, Tung NH, Morinaga O, Shoyama Y (2011) Preparation of knockout extract by immune affinity column and its application. Antibodies 1: 294-307.

32. Peng F, Du Q, Peng C, Wang N, Tang H, et al. (2015) A Review: The Pharmacology of Isoliquiritigenin. Phytother Res 29: 969-977. 